\title{
Stimulus context and duration discrimination
}

\author{
DAVID D. WOODS, ROBERT D. SORKIN, and GEORGE J. BOGGS \\ Purdue University, West Lafayette, Indiana 47907
}

\begin{abstract}
The ability of human observers to discriminate duration was assessed in two types of tasks: (1) pulse tasks, in which the observer compared the duration of two brief increments in an ongoing sinusoid, and (2) gap tasks, in which the observer compared the duration of two brief interruptions in an ongoing sinusoid. Performance in these tasks was assessed in three different contexts: noise alone, noise plus continuous sinusoids, and noise plus continuous sinusoids chosen to induce a pitch segregation effect. Performance in the pulse task was independent of the changes in context; however, performance in the gap task changed as a function of context condition. There was a large decrement in the observers' ability to discriminate duration when the stimulus ensemble induced the pitch segregation effect. The results are discussed in terms of the relationship between duration discrimination and stimulus variations which do not carry duration information.
\end{abstract}

Allan and Kristofferson (1974) have made a convincing argument that duration discrimination involves processes fundamentally different from those involved in discrimination along other stimulus dimensions, such as amplitude and frequency. They stress three points: (1) duration discrimination is relatively independent of the temporal and intensive parameters of the stimuli which bound or fill the judged intervals, (2) duration discrimination is independent of the interstimulus interval between sequentially presented intervals, and (3) there is no apparent performance difference between forced-choice and single-interval duration discrimination tasks. We are primarily concerned with the generality of point 1 : To what extent does duration discrimination depend on stimulus variations which do not signal duration differences?

Traditionally, investigators of duration discrimination have tried to minimize cues derived from the spectral consequences of changes in the duration of tones, since those cues might provide a basis for task performance. The present study will show that an observer's ability to discriminate auditory durations is very sensitive to changes in the stimulus context, even when the influence of spectral and energetic factors has been minimized.

Allan and Kristofferson (1974) reviewed many duration discrimination studies and concluded that performance is unaffected by variations in intensity for filled visual intervals (Allan, Kristofferson, \& Wiens, 1971) and for filled auditory intervals (Abel, 1972a, for noise bursts; Creelman, 1962, for tone signals). Similarly, changes in the duration and the intensity

This research was supported by National Science Foundation Grant BNS-77-13503. Reprints may be obtained from R. D. Sorkin, Department of Psychological Sciences, Purdue University, West Lafayette, Indiana 47907. of the markers of empty intervals do not produce changes in judgments of temporal extent as long as the markers are clearly detectable (Abel, 1972b; Carbotte \& Kristofferson, 1973; Divenyi \& Danner, 1977, for unfilled auditory intervals; Nilsson, 1969, for unfilled visual intervals). Abel (1972b) did report a small decrease in the $\Delta t$ necessary for $75 \%$ correct discriminations in a 2AFC task as the amplitude of noise markers decreased from 85 to $70 \mathrm{~dB}$ SPL. However, Carbotte and Kristofferson (1973) and Divenyi and Danner (1977) varied marker intensity over larger ranges and found no changes in discrimination performance. ${ }^{1}$

In general, changes in the intensive parameters of stimuli that signal duration do not affect an observer's ability to discriminate time intervals. However, duration discrimination performance is sensitive to some aspects of the stimulus ensemble. For example, Penner (1976) found that randomizing the duration and intensity of noise burst markers greatly increased the $\Delta t$ necessary for $75 \%$ correct performance compared to fixed marker control conditions for a variety of base durations. Randomizing marker parameters can be considered as an increase in task uncertainty, since the observer does not know the exact stimulus that will be presented on any given trial.

Divenyi and Danner (1977) found that frequency differences between the tones used to mark empty intervals can produce large (fourfold) decrements in temporal resolution. Collyer (1974) and Perrott and Williams (1971) have also reported elevated temporal thresholds when different frequency tones were used to signal duration. Williams and Perrott (1972) found that increasing the duration of the marker tones increased the effect of frequency differences on duration discrimination. On the basis of these studies, Divenyi and Danner concluded that spectral and intensive factors do not affect duration discrimination as long 
as the stimuli that carry the temporal information are processed in the same channel.

When observers are asked to judge the duration of a temporal interval, the pattern of stimulation can affect performance. Thomas and Brown (1974) examined the effects of filler events on the discrimination of temporal gaps and found that filled intervals are perceived as longer in duration than are empty intervals. This filled-duration illusion occurs across different kinds of filler events: discrete events (for example, a series of clicks, Thomas \& Brown, 1974), continuous stimulation (Craig, 1973), and more complex stimuli such as interpolated mathematical tasks (Burnside, 1971). The filled-duration effect implies that the presence of stimuli that change the overall stimulus context can greatly affect the perception of temporal intervals.

The processing of duration information appears to be independent of many kinds of changes in the spectral and intensive characteristics of the stimuli used to carry the duration information. However, the effects of channel, marker uncertainty, and the pattern and context of stimulation, suggest that cognitive factors may influence the timing mechanism even though peripheral interactions do not. Massaro and Idson (1978), in a backward masking (of duration) paradigm, concluded that the time available to process duration information affects the perceived duration. This effect appeared to be the result of processing beyond the level of peripheral interactions, since it was independent of the frequency of the masker.

Kubovy (Note 1) has demonstrated a subjective effect, which he called pitch segregation, that we believed could be useful for investigating the interaction of nonspectral context effects and duration discrimination. Given a group of ongoing tones, a momentary interruption (for 20 to $100 \mathrm{msec}$ ) in one of the tones causes the pitch of the interrupted tone to perceptually dominate the chord for an extended period of time.

The phenomenal effect is to hear a tone that has the same pitch as the sinusoid that has been interrupted, but for a duration which far exceeds the actual duration of the interruption. The interrupted tone appears to stand out for a long period of time as a foreground against the background of the other sinusoids. It is important to note that interrupting one of a set of tones results in a clearly different percept than momentarily adding an increment to one of the sinusoids that is the same size and duration as the interruption (e.g., doubling the amplitude). A particular spectral context is needed to produce this subjective pitch effect; removing all tones except the interrupted one eliminates the persistent pitch associated with the interrupted sinusoid. The frequency relationships within the group of continuous tones are also important. As the frequency sep- aration between the sinusoids is decreased, the salience of the effect decreases and then disappears. A significant aspect of Kubovy's demonstration is that the subjective effect greatly exceeds the duration of any spectral consequences of briefly turning off and on a sinusoid. That is, whatever the origin of the pitch segregation effect, it may be used to induce a perceptual consequence which has no immediate spectral or energetic correlate.

We asked observers to discriminate durations that were displayed as increments in an ongoing sinusoid (pulse conditions) and also to discriminate durations that were displayed as interruptions in an ongoing sinusoid (gap conditions). Three variations of these two tasks were run: (1) a condition where the only background was white noise, (2) a condition where the background consisted of white noise plus two continuous sinusoids at frequencies slightly more than one critical band above and below the frequency of the gapped or pulsed tone, and (3) a condition where the background was white noise plus two ongoing sinusoids much more than one critical band above and below the frequency of the gapped or pulsed tone. We chose the frequencies of the sinusoidal sidebands to yield either no subjective pitch segregation effect (condition 2) or a strong subjective pitch segregation effect (condition 3 ) when the middle frequency tone was interrupted. These three conditions form a set of manipulations which allow some specification of the influence of nonspectral context on duration discrimination. Note that in the pulse conditions the time discrimination is based on a filled interval (the duration of an increment in a sinusoid), and in the gapped cases the discrimination is based on a temporal gap in one sinusoid.

While the pulse and gap conditions represent different tasks, we can evaluate differences in the pattern of results for these two tasks across the three context conditions. For example, changes in the slope of the discrimination performance vs. $\Delta t$ function should indicate process differences in the different tasks. Since the sidebands are always at least a critical band away from the transient events of interest and since they are fixed within a block of trials, there is little or no spectral or energetic basis to expect performance differences across the three context conditions. If there is any spectral masking between the continuous sinusoids and the gap/pulse event, performance would be worse in case 2 , where the side tones are closest in frequency. An interaction of the subjective pitch effect with duration discrimination would result in poorest performance in condition 3, but only in the gapped condition.

\section{METHOD}

Data was collected from three female observers who were paid an hourly rate plus a bonus based on their performance. Two 
of the subjects were graduate students in the Purdue Department of Audiology and Speech Sciences (observers B and G) and had had extensive exposure to psychoacoustic tasks in their program of study. The third observer (L) was an undergraduate with no previous experience at psychophysical tasks. Stimuli were presented over TDH-39 headphones, and were band-limited only by the frequency response of the headphone; observers worked inside a single-walled Industrial Acoustics chamber within a separate acoustically insulated room. All stimuli were presented monaurally to the observer's right ear. The timing and presentation of the stimuli and the recording of data was controlled by a PDP $-8 / \mathrm{L}$ minicomputer.

The experimental paradigm was a two-interval forced-choice task. The observers had to choose whether the first or second interval contained the longer duration stimulus. The two intervals differed only in the duration of the events of interest, and the longer duration stimulus could appear in either interval with equal probability. Thus, there were two kinds of trials: $t, t+\Delta t$ or $t+\Delta t$, $t$. The base duration $(t)$ was always $40 \mathrm{msec} ; \Delta t$ varied depending on the observer and condition from 5 to $50 \mathrm{msec}$. On a given trial, the observer always heard a low-level, continuous white Gaussian noise at a spectrum level of $33 \mathrm{~dB} / \mathrm{Hz}$ and a continuous $1,000-\mathrm{Hz}$ sinusoid at $71 \mathrm{~dB}$ SPL. In the pulse conditions, the observers based their duration judgments on the duration of the two $6-\mathrm{dB}$ increments in the $1,000-\mathrm{Hz}$ tone; in the gap conditions, they judged the durations of two total interruptions in the $1,000-\mathrm{Hz}$ tone. The increments and interruptions had a rise and decay time of $1 \mathrm{msec}$.

Three context conditions were formed by (1) adding no extra tones, (2) by adding two side tones at 810 and $1,335 \mathrm{~Hz}$, or (3) by adding two side tones at 680 and $1,790 \mathrm{~Hz}$. All tones used were presented at a level of $71 \mathrm{~dB}$ SPL. Only in context condition 3 did an interruption in the $1,000-\mathrm{Hz}$ tone produce the pitch segregation effect. Trial events consisted of a $200-\mathrm{msec}$ warning light $200 \mathrm{msec}$ before the first observed duration. One second after the first pulse or gap ended, the second occurred. The trial sequence then paused until the observer made a response, provided observer feedback, and paused for $500 \mathrm{msec}$ before the next trial.

All three observers had extensive experience with the pitch segregation effect in a different psychophysical task before they participated in this experiment. The observers first ran 2 days on a gap-based duration discrimination task and then 2 days on a pulsebased duration discrimination task. Observers ran 900 trials per day. All three observers found the gap-based task more difficult; therefore, the $\Delta t$ for gap conditions was longer than that for the pulse conditions. Each context condition was run each day for 300 trials in the following order: noise-only context, $810-1,335-\mathrm{Hz}$ sideband contexts, $680-1,790-\mathrm{Hz}$ sideband context. In a second phase of the experiment, two new values of $\Delta t$ were used for each condition in order to determine three-point psychometric functions. Only observers $B$ and $G$ participated in this phase. In summary, there were six conditions, each run at three $\Delta t$ values: two types of duration discrimination (gap or pulse) by three kinds of context (noise only, $810-1,335-\mathrm{Hz}$ sidebands, or 680-1,790- $\mathrm{Hz}$ sidebands).

\section{RESULTS}

Table 1 summarizes the results from the first phase of the experiment. The table entries consist of $d^{\prime}$ scores with the corresponding $\beta$ s in parentheses. These scores were computed by considering "first interval longer" responses when the first interval did contain the longer stimulus $\left(R_{1} / I_{1}\right)$ as hits, and "first interval longer" responses when the second interval contained
Table 1

Observer Performance on the Two Types of Task Across Context Conditions

\begin{tabular}{ccccc}
\hline & & \multicolumn{3}{c}{ Context Condition } \\
\cline { 3 - 5 } Task & Subject & Noise Only & $\begin{array}{c}810- \\
1,335-\mathrm{Hz}\end{array}$ & $\begin{array}{c}680- \\
1,790-\mathrm{Hz}\end{array}$ \\
& & & & \\
Sidebands & Sidebands \\
\hline \multirow{4}{*}{ Pulse } & $\mathrm{B}(\Delta \mathrm{t}=20)$ & $1.82(1.08)$ & $1.93(1.02)$ & $2.20(1.45)$ \\
& $\mathrm{G}(\Delta \mathrm{t}=20)$ & $2.02(1.10)$ & $1.67) 1.12)$ & $1.77(1.18)$ \\
& $\mathrm{L}(\Delta \mathrm{t}=30)$ & $1.43(1.12)$ & $1.17(1.29)$ & $1.52(1.19)$ \\
& $\mathrm{B}(\Delta \mathrm{t}=40)$ & $1.93(1.06)$ & $.94(1.09)$ & $.27(1.00)$ \\
Gap & $\mathrm{G}(\Delta \mathrm{t}=30)$ & $1.71(1.05)$ & $1.32(1.19)$ & $.14(1.00)$ \\
& $\mathrm{L}(\Delta \mathrm{t}=40)$ & $1.30(.89)$ & $1.03(.88)$ & $.79(.87)$ \\
\hline
\end{tabular}

Note-Table entries are $d$ 'scores with the corresponding $\beta$ in parentheses.

the longer stimulus $\left(R_{1} / I_{2}\right)$ as false alarms. This method of calculating $\mathrm{d}^{\prime}$ was used since no observer demonstrated any appreciable bias to consistently prefer one response over the other (all criterion measures were close to one).

In the pulse-based duration discrimination task, there were only small and inconsistent differences in sensitivity across the different contexts. However, when a gap rather than a pulse was used to mark duration, there was a clear effect of context on sensitivity. The presence of any extraneous sinusoids, even though they were at least a critical bandwidth away from the frequency of interest, resulted in a decrease in performance. Furthermore, this decrement was considerably larger when the sideband frequencies were 680 and $1,790 \mathrm{~Hz}$-the context condition designed to produce the subjective pitch effect. All three observers showed this pattern of results. Adding sidebands at 810 and $1,335 \mathrm{~Hz}$ produced an average performance decrement of $.53 \mathrm{~d}^{\prime}$ units. If the sidebands were placed instead at 680 and $1,790 \mathrm{~Hz}$, then the decrement averaged $1.23 \mathrm{~d}^{\prime}$ units. These differences are large compared to the expected standard deviations for the data points in Table 1 (assuming a binomial distribution) which are less than $.2 \mathrm{~d}^{\prime}$ units (Green \& Swets, 1966).

Three-point psychometric functions were computed from the different $\Delta t$ values within each condition. These functions are displayed in Figures 1 and 2 for the two observers (B and G, respectively) who participated in this part of the experiment. The results can be seen to be consistent with those from the first phase of the analysis. Performance was relatively constant across context condition with the pulse task. However, when a gap marked duration, the presence of extraneous tones decreased performance, particularly in the $680-1,790-\mathrm{Hz}$ sideband condition. The slopes of the psychometric functions were consistent across observer, task type, and context condition, 


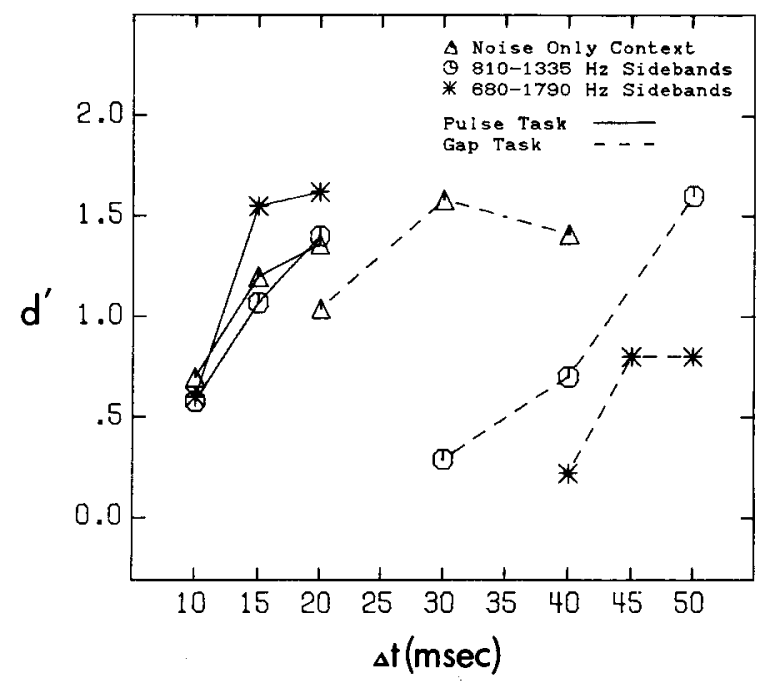

Figure 1. Observer B's performance (d') as a function of $\Delta t$ for the pulse and gap tasks and the three context conditions.

except for observer $\mathrm{G}$ in the gap-based task and the $810-1,335-\mathrm{Hz}$ context condition.

\section{DISCUSSION}

The pattern of results across context condition is clearly different in the gap and pulse tasks. When duration information is marked by the absence of the signal, performance is highly sensitive to the characteristics of surrounding events. Duration discrimination appears to involve different processes when the intervals to be judged are filled rather than empty. This is a curious state of affairs since using unfilled intervals or gaps in stimuli was originally proposed in order to minimize potential spectral and intensive cues that could aid discrmination performance (Abel, 1972b). Instead, these paradigms seem to provide greater opportunity for contextual factors to influence duration discrimination.

Inserting continuous sinusoids, at frequencies more than a critical band away from the events of interest, should not affect the observers' ability to discriminate duration. Since the context-determining tones do not change during an experimental trial or a block of trials, they should not divert attention from the region of interest. Since these tones are far removed in frequency from the to-be-discriminated events, there should be no energetic interactions. Indeed, if there are any interactions, they should increase, not decrease, as the sideband tones converge in frequency. However, the frequency relationships in the $680-1,790-\mathrm{Hz}$ context condition induce a qualitative change-a persisting pitch. Even though there are no significant intensive or spectral differences between these context conditions, our results clearly show that a qualitative change in how the stimuli are perceived changes duration discrimination perfor- mance. This effect is probably mediated through changes in the perceived relationship between the time markers and the background.

We stated earlier in a description of the pitch segregation effect that it produces a pitch that persists longer than the actual interruption used to produce it. This conjecture, based simply on listening to the effect, is consistent with our results. Since the perceived duration in the subjective pitch condition exceeds the actual event duration, the decrease in discrimination performance could be due to a Weber's law effect. Increasing the subjective base duration of the stimulus would require increasing $\Delta t$ to maintain constant performance. Also note that while the physical basis of the time information in the subjective pitch condition consists of an interruption in a sinusoid, the subjective result is the perception of an event-a persisting pitch. Thus, perceptually, the task in the pitch segregation condition may consist of discriminating the durations of filled rather than empty intervals.

The original point of departure for this work was the observation of Allan and Kristofferson (1974) that duration discrimination is independent of the spectral and intensive characteristics of the stimuli used to mark duration. Collyer (1974) and Rousseau and Kristofferson (1973) reasoned that independently processed duration markers would eliminate any nontemporal basis for performing the task. In order to test this idea, they investigated the discrimination of empty intervals marked by bimodal stimuli. Collyer's results indicated a large decrement in performance when bimodal, rather than intramodal, duration markers were used. Increasing the frequency difference between tone markers should also minimize peripheral interactions, since the stimuli would be

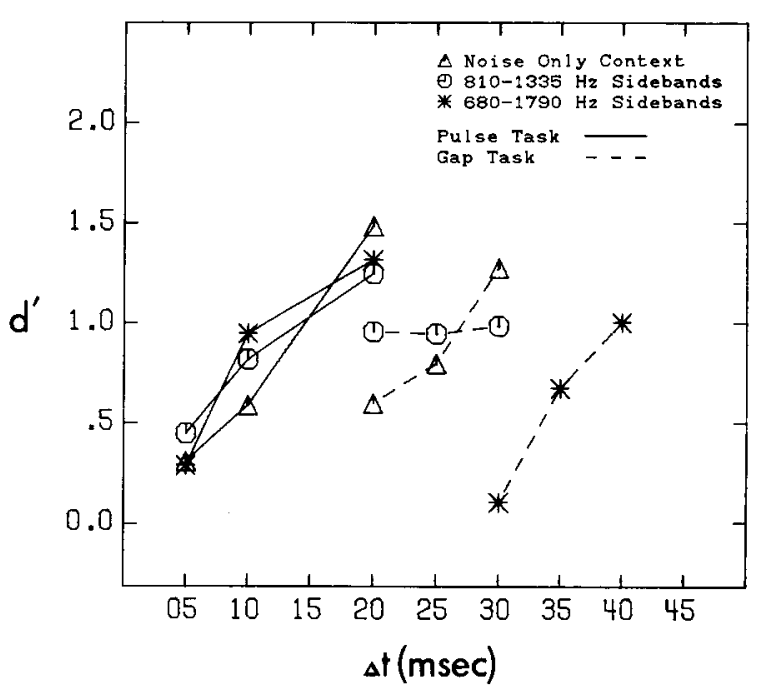

Figure 2. Observer G's performance ( $d^{\prime}$ ) as a function of $\Delta t$ for the pulse and gap tasks and the three context conditions. 
processed in different frequency channels. Collyer's data, as well as results from Divenyi and Danner (1977) and Perrott and Williams (1971), show that increasing the frequency separation between tonal markers also decreases duration-discrimination performance.

While this decrease in performance could be due to the elimination of extra cues derived from peripheral interactions, there is an alternative explanation. Presenting marker stimuli in different channels might introduce new variables into the task-channel uncertainty and channel switching time. These highlevel factors may be responsible for the decrease in an observer's ability to discriminate duration.

Experiments that have explored the characteristics of auditory stream segregation imply a similar result. Temporal resolution is severely limited across perceptual streams formed on the basis of frequency differences (Bregman \& Campbell, 1971). Fitzgibbons, Pollatsek, and Thomas (1974) asked observers to detect a gap in a four-tone sequence. The sequence was grouped into two perceptual streams by choosing one pair of tones from a low-frequency set and the second pair from a high-frequency set. The independent variable was the placement of the gap within the sequence. The gap was considerably harder to detect when it occurred at a frequency transition. This could be because time is needed to switch attention between perceptual streams, as Fitzgibbons concluded, or because the stream segregation phenomenon rearranges the temporal relationships within the sequence. The fact that observers "invariably" localized the gap at the frequency transition when the gap was actually located between two tones in the same stream supports the latter view. Fitzgibbons used the same basic paradigm as Collyer, except that a sequence of tones rather than single tones was used to mark the to-be-detected gap. Since Fitzgibbons did not run a single tone marker control, we cannot determine whether the frequency difference alone or the perceptual streams, or both, produced the decrease in detectability. Nevertheless, all of the above studies imply that there is some loss of temporal resolution when the events that signal duration are processed in different channels-either different frequency channels, different perceptual streams, or different modalities.

We must agree with Allan and Kristofferson's (1974) position that duration discrimination involves fundamentally different processes than does discrimination along other stimulus dimensions. We must also agree that performance on duration discrimination tasks is essentially independent of the temporal, intensive, and spectral characteristics of the events that mark duration, as long as they are clearly detectable and processed in the same channel. However, the perception of duration can be affected by such factors as stimulus uncertainty, multichannel presentation, and the time available to process the duration information. These effects imply that higher level, nonperipheral factors can interact with the processing of duration information. The present study clearly supports this conclusion. The perceived duration of events is influenced by the stimulus context, the pitch segregation effect, even though this context produces no significant spectral or energetic changes in the stimulus.

\section{REFERENCE NOTE}

1. Kubovy, M. The sound of silence: $A$ new pitch segregation phenomenon. Paper presented at the meeting of the Psychonomic Society, St. Louis, Missouri, November 1976.

\section{REFERENCES}

Abel, S. M. Duration discrimination of noise and tone bursts. Journal of the Acoustical Society of America, 1972, 51, 1219-1223. (a)

AвEL, S. M. Discrimination of temporal gaps. Journal of the Acoustical Society of America, 1972, 52, 519-524. (b)

Allan, L. G., \& Kristofferson, A. B. Psychophysical theories of duration discrimination. Perception \& Psychophysics, 1974, 16, 26-34.

Allan, L. G., Kristofferson, A. B., \& Wiens, E. W. Duration discrimination of brief light flashes. Perception \& Psychophysics, $1971,9,327-334$.

Bregman, A. S., \& Campbell, J. L. Primary auditory stream segregation and perception of order in rapid sequences of tones. Journal of Experimental Psychology, 1971, 89, 244-249.

Burnside, W. Judgment of short time intervals while performing mathematical tasks. Perception \& Psychophysics, 1971, 9, 404-406.

Carbotte, R. M., \& Kristofferson, A. B. On energy-dependent cues in duration discrimination. Perception \& Psychophysics, 1973, 14, 501-505.

Collyer, C. E. The detection of a temporal gap between two disparate stimuli. Perception \& Psychophysics, 1974, 16, 96-100.

Craig, J. C. A constant error in the perception of brief temporal intervals. Perception \& Psychophysics, 1973, 13, 99-104.

Creelman, C. D. Human discrimination of auditory duration. Journal of the Acoustical Society of America, 1962, 34, 582-593.

DivenYi, P. L., \& Danner, W. F. Discrimination of time intervals marked by acoustic pulses of various intensities and spectra. Perception \& Psychophysics, 1977, 21, 125-142.

Fitzgibeons, P. J., Pollatsek, A., \& Thomas, I. B. Detection of temporal gaps within the between perceptual tonal groups. Perception \& Psychophysics, 1974, 16, 522-528.

Green, D. M., \& Swets, J. A. Signal detection theory and psychophysics. New York: Wiley, 1966.

Massaro, D. W., \& IDson, W. L. Target-mask similarity in backward recognition masking of perceived tone duration. Perception \& Psychophysics, 1978, 24, 225-236.

Nissson, T. H. Two-pulse-interval vision thresholds. Journal of the Optical Society of America, 1969, 59, 753-757.

Penner, M. J. The effect of marker variability on the discrimination of temporal intervals. Perception \& Psychophysics, 1976, 19, 466-469.

Perrott, D. R., \& Williams, K. N. Auditory temporal resolution: Gap detection as a function of interpulse frequency disparity. Psychonomic Science, 1971, 25, 73-74.

Pollack, I. Detection and relative discrimination of auditory 
"jitter." Journal of the Acoustical Society of America, 1968, 43, 308-315. (a)

Pollack, I. Discrimination of mean temporal interval within jittered auditory pulse trains. Journal of the Acoustical Society of America, 1968, 43, 1107-1112. (b)

Rousseau, R., \& Kristofferson, A. B. The discrimination of bimodal temporal gaps. Bulletin of the Psychonomic Society, 1973, 1, 115-116.

Thomas, E. A. C., \& Brown, J. Time perception and the filled duration illusion. Perception \& Psychophysics, 1974, 16, 449-458.

Williams, K. N., \& Perrott, D. R. Temporal resolution of tonal pulses. Journal of the Acoustical Society of America, $1972,51,644-647$.

\section{NOTE}

1. There are specific situations in audition where observers can distinguish monaurally presented stimulus variations in the microsecond range. These extremely fine discriminations seem to be based on a spectral analysis of the input (Pollack, 1968a, 1968b). The present discussion is restricted to time discriminations in the millisecond range.
(Received for publication March 12, 1979; revision accepted June 4, 1979.) 